\title{
Pancreatic Cancer with Normal CA 19-9 and Lewis
}

\section{Antigen Negative-Case Report}

\author{
Rodrigo Coutinho Mariano ${ }^{1}$, Noam Fabel Ponde ${ }^{2}$, Daniel Eiger ${ }^{1}$, Patricia Taranto ${ }^{1}$, Vanderlei Segatelli ${ }^{1}$, Daniela \\ Pezzutti Armentano ${ }^{1}$ and Rene Claudio Gansl ${ }^{1}$ \\ 1. Hospital Israelita Albert Einstein, São Paulo 627, Brazil \\ 2. Jules Bordet Institute, Brussels 1000, Belgium
}

\begin{abstract}
Pancreatic cancer is an aggressive and lethal disease that affects especially older population. Its more relevant tumor marker is CA 19-9 (carbohydrate antigen 19-9), although it can be elevated in others clinical situations, like cholangitis and cholestasis. Otherwise, a small people subset, like our patient, do not produce this tumor marker, as on blood as in the tumor, because they are incapable to express the Lewis Antigen. Therefore, this case report is about a patient without Lewis Antigen express and CA 19-9 low levels. We will report a rapid disease progression, despite of low CA 19-9, comparing with available data that often show better prognosis in this setting. Conclusion: Low levels of CA 19-9 do not predict good response or better prognosis in patients that do not express Lewis Antigen.
\end{abstract}

Key words: Pancreatic cancer, CA 19-9, Lewis Antigen, prognosis.

\section{Introduction}

Pancreatic cancer, more prevalent in older population, (mean age of 71 year-old), is known as a very aggressive and lethal disease. It affects around 46,000 new cases per year in USA and its mortality specific-disease is around 40,000 people per year. The most common histology is ductal adenocarcinoma, which correspond to $85 \%$ of the cases [1].

CA 19-9 is the most important and common tumor marker in pancreatic cancer, with sensitivity and specificity of $70 \sim 92 \%$ and $68 \sim 92 \%$, respectively [2-7]. This protein can also be elevated in benign setting, like cholangitis and cholestasis [8-10].

Otherwise, this tumor marker has a low positive predictive value and this is the reason to not consider this as a good diagnostic tool [10]. Furthermore, the sensibility can change based in some tumor features, like tumor burden, and phenotypic expression of Lewis Antigen.

Patients unable to express Lewis antigen (due to lack

Corresponding author: Rodrigo Coutinho Mariano, M.D., research fellow, research field: medical oncology. of functional FUT3 allele) are unable to produce CA 19-9, as well [8-12]. This condition affects 5 10\% of general population $[8,9,11]$. However, a prospective study that assessed the role of CA 19-9 as a prognostic marker in post-operatory patients with pancreatic cancer found $34 \%$ patients with Lewis Antigen negative. This was attributed to some differences between methods used in assessments [11].

Furthermore, CA 19-9 has a questionable role as chemotherapy response predictive factor, but a well establish role in predict resecability [12-17], prognosis and in follow-up of these patients [7-12].

In view of this, our objective is to show a case report of a pancreatic adenocarcinoma in a patient without Lewis Antigen express and low grades of CA 19-9, to assess and compare outcomes and biological behavior of this condition with available data, and arise further questions about the real role of this tumor marker in this setting.

\section{Methods}

This case report was based on appointment with patient and information written in her profile. 
Photograph records of diagnostic methods were used, as well. Tumor features and outcomes were assessed and compared with available based literature data.

\section{Case Report}

An 87 year-old female patient demanded medical care due to severe abdominal pain in upper-right side with back irradiation. She has complained prostration and loss of appetite for two months. Her physical exam was unremarkable.

She had a Stage-II duodenal adenocarcinoma (fourth portion) 10 years before which was surgically treated, margins free. She had started follow-up without relapses throughout this period. Some suspicious cystic lesions were viewed and the hypothesis was (IPMN) intraductal papillary mucinous neoplasia. She denied familial history for cancer.

The patient underwent an abdominal MRI (magnetic resonance imaging) that showed several ovals lesions, greater than $5.0 \mathrm{~cm}$, thin and regular walls, suggestive of cystic lesions. Furthermore, four hepatic nodules were viewed up to $2.7 \mathrm{~cm}$ in major axis, suggesting secondary lesions.

Therefore, tumor markers were asked (CEA (carcinoembryonic antigen), CA 15-3, CA 125 and CA 19-9) and everyone was at higher levels, except for CA 19-9. It was below of lower limits of normal (CA 19-9 = 2.12).

Thus, the patient underwent a tomography-guided biopsy of one hepatic lesion. Microscopic evaluation of biopsy revealed metastatic adenocarcinoma with areas of necrosis (Fig. 1a). The immunohistochemical study showed positive immunoreactivity for cytokeratin 7 and 19 (Fig. 1b) and positive variably for cytokeratin 20, CDX2 and GATA3 and negative immunostaining for CA 19-9 (Fig. 1c), estrogen receptor and TTF1 (thyroid transcription factor 1).

After this, a CT-PET scan (Fig. 2) was performed and showed uptake in pancreatic tail, hepatic nodules, hepatic hilum and retroperitonium lymph nodes were observed. Further, uptake in pulmonary nodules and mediastinal lymph nodes were be observed, suggesting secondary lesions either.

Given this uncommon CA 19-9 low levels presentation in a probably pancreatic cancer, we decided to ask Lewis antigen erythrocyte fenotipic. The result, as well expected, was negative for expression.

After clinical and radiologic evaluations, we opted to start first line palliative chemotherapy with Gencitabine. Three completes cycles was performed, with some adjusts due to hematological toxicity. In the first re-staging, patient was clinically worse. He had PD (progressive disease) in primary site (Fig. 3), liver, lung and adrenal. Furthermore, CEA increased but CA 19-9 was still low. Hence, chemotherapy was interrupted and exclusively best supportive of care was initiated.

After some weeks, patient was admitted at the hospital with worsing of level of consciousness due to hepatic encephalopathy, evolving to death.

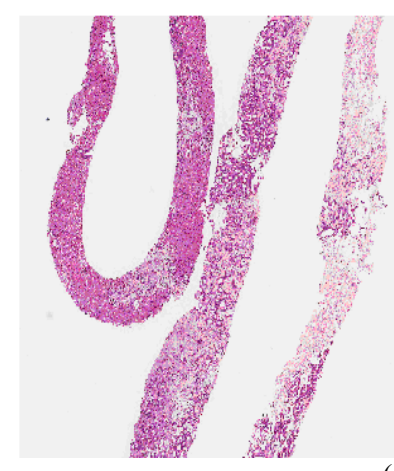

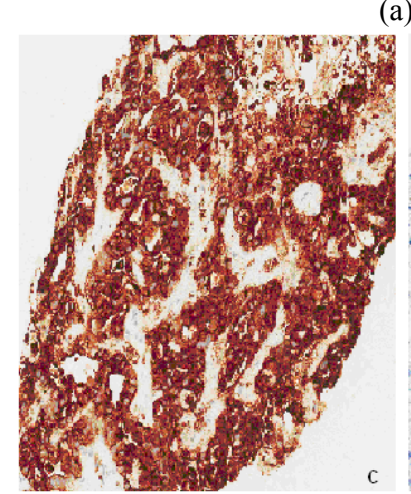

(b)

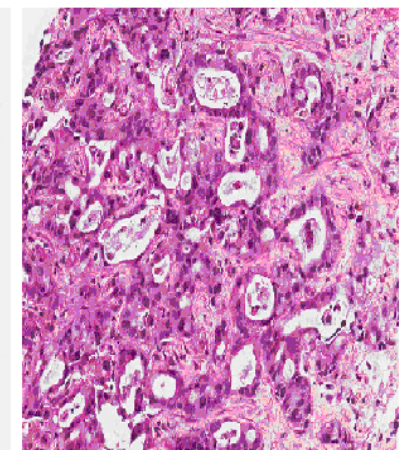

(a)

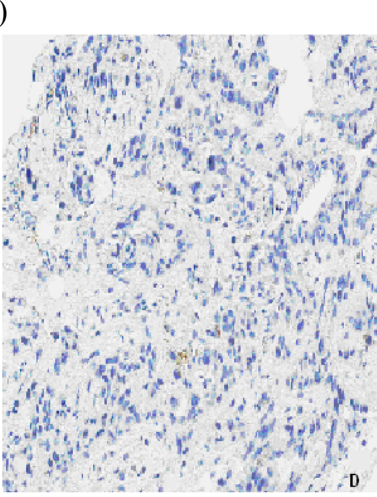

(c)
Fig. 1 Microscopic evaluation: (a) liver biopsy showing metastatic adenocarcinoma with areas of necrosis; (b) diffuse positive immunostaining for cytokeratin 19; (c) negative immunoreactivity for CA 19.9 in neoplastic cells. 


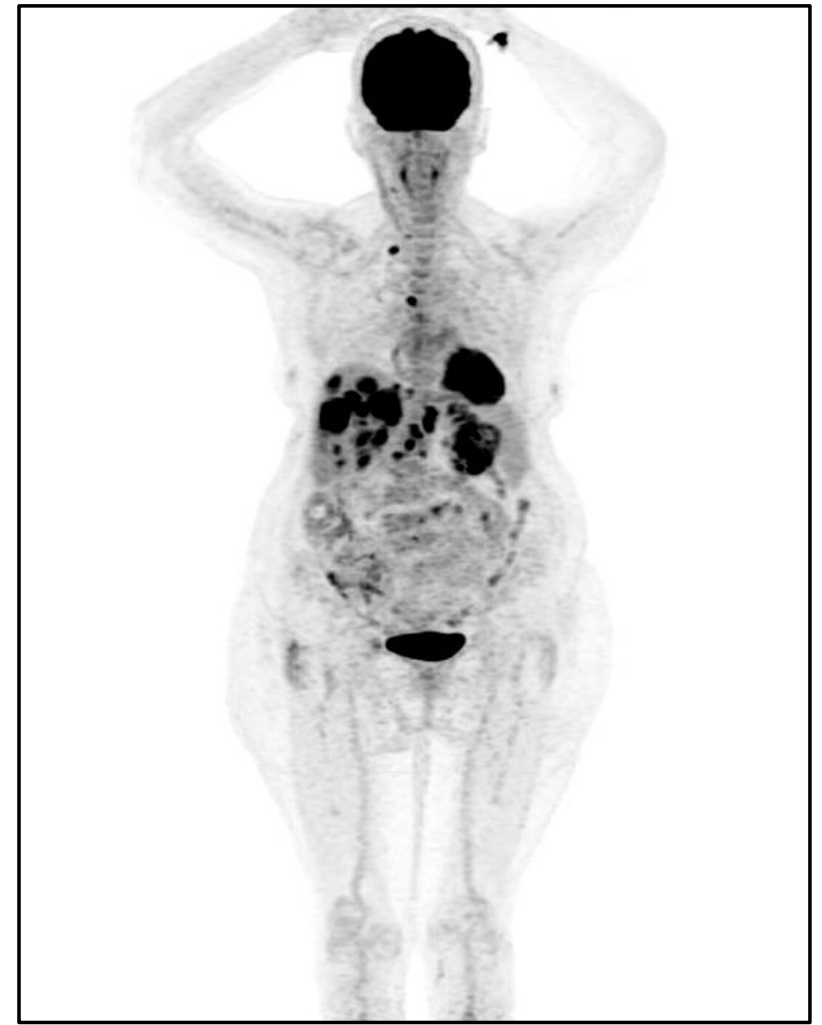

Fig. 2 CT-PET scan showing multiples uptake sites, including pancreatic tail.

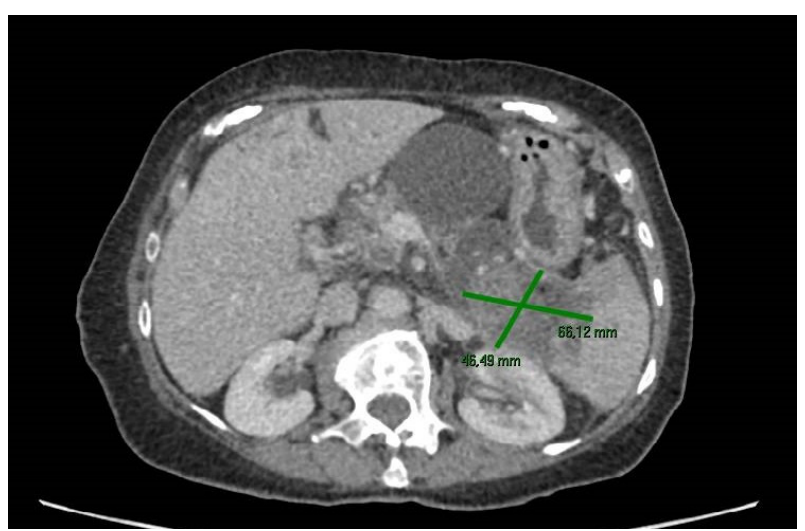

Fig. 3 Abdominal CT after third chemotherapy cycle with gemcitabine.

\section{Discussion}

As showed above, pancreatic cancer natural behavior presents high mortality rates, mainly in extensive metastatic setting [1]. This reported patient died after just four months from her diagnosis. This was not expected in cases like her whom CA 19-9 was low. The RTOG 9704 trial showed these patients who did not express Lewis Antigen usually have better prognostic and outcomes than people with high levels (greater than $90 \mathrm{U} / \mathrm{mL}$ ) [7]. Furthermore, others available data often show better prognosis when CA 19-9 is low [12-13].

On the other hand, some others prognostic factors probably also influenced this case, like presence of hepatic and lymph node disease [1, 7, 18, 19]. Furthermore, cancer developing from IPMN often has worse behavior and can be another explanation to this catastrophic outcome [1].

Others prognostic factors in pancreatic cancer also have been described, like histology grade, lymph-vascular or neural invasion, tumor burden, commorbities, prior adjuvant therapy or not, margins status [19].

\section{Conclusions}

Therefore, our case reported a patient with pancreatic cancer without Lewis Antigen expression (5 10\% of people) and CA 19-9 low as well. However, outcomes were catastrophic, very different we expected based on literature evidences showing a better prognosis when CA 19-9 is low. This fact arises some questions about real role as a prognostic factor in patients that cannot express it. Then, others variants, like previous IPMN and metastatic disease at diagnosis, might have more relevance in predict prognosis in this specific patients' group.

\section{References}

[1] Ryan, D. P., Hong, T. S., and Bardeesy, N. 2014. "Pancreatic Adenocarcinoma." N. Engl. J. Med. 371: 1039-49.

[2] Pleskow, D. K., Berger, H. J., Gyves, J., Allen, E, McLean, A., and Podolsky, D. K. 1989. "Evaluation of a Serologic Marker, CA19-9, in the Diagnosis of Pancreatic Cancer." Ann. Intern. Med. 110 (9): 704-9.

[3] Cwik, G., Wallner, G., Skoczylas, T., Ciechanski, A., and Zinkiewicz, K. 2006. "Cancer Antigens 19-9 and 125 in the Differential Diagnosis of Pancreatic Mass Lesions." Arch. Surg. 141 (10): 968-73.

[4] Van den Bosch, R. P., Van Eijck C. H., Mulder P. G., and Jeekel, J. 1996. "Serum CA 19-9 Determination in the Management of Pancreatic Cancer." 
Hepatogastroenterology 43 (9): 710-3.

[5] Paganuzzi, M., Onetto, M. ,Marroni P., Barone D., Conio M., Aste H., et al. 1998. "CA 19-9 and CA 50 in Benign and Malignant Pancreatic and Biliary Diseases." Cancer 61 (10): 2100-8.

[6] Malesci, A., Tommasini, M. A., Bonato, C., Bocchia, P., Bersani, M., Zerbi, A., et al. 1987. "Determination of CA 19-9 Antigen in Serum and Pancreatic Juice for Differential Diagnosis of Pancreatic Adenocarcinoma from Chronic Pancreatitis." Gastroenterology 92 (1): 60-7.

[7] Berger, A. C., Garcia, M. Jr., Hoffman, J. P., Regine, W. F., Abrams, R. A., Safran, H., et al. 2008. "Postresection CA 19-9 Predicts Overall Survival in Patients With Pancreatic Cancer Treated With Adjuvant Chemoradiation: A Prospective Validation by RTOG 9704." J. Clin. Oncology 26 (36): 5918-22.

[8] Lamerz, R., 1999. "Role of Tumour Markers, Cytogenetics." Ann. Oncol. 10 (4): 145-9.

[9] Goggins, M. 2005. "Molecular Markers of Early Pancreatic Cancer.” J. Clin. Oncol. 23 (20): 4524-31.

[10] Kim, H. J., Kim, M. H., Myung, S. J., Lim, B. C., Park ,E. T., Yoo, K. S., et al. 1999. "A New Strategy for the Application of CA 19-9 in the Differentiation of Pancreatic-Biliary Cancer: Analysis Using a Receiver Operating Characteristic Curve.” Am. J. Gastroenterol 94 (7): 1941-6.

[11] Tempero, M. A., Uchida, E., Takasaki, H., Burnett, D. A., Steplewski, Z., and Pour, P. M. 1987. "Relationship of Carbohydrate Antigen 19-9 and Lewis Antigens in Pancreatic Cancer." Cancer Res. 47 (20): 5501-3.

[12] Maisey, N. R., Norman, A. R., Hill, A., Massey, A., Oates, J., and Cunningham, D. 2005. "CA 19-9 as a Prognostic Factor in Inoperable Pancreatic Cancer: The Implication for Clinical Trials.” Br. J. Cancer 93 (7): 740-3.

[13] Humphris, J. L., Chang, D. K., Johns, A. L., Scarlett, C. J., Pajic, M., Jones, M. D., et al. 2012. "The Prognostic and Predictive Value of Serum CA 19.9 in Pancreatic Cancer." Ann. Oncol. 23 (7): 1713-22.

[14] Maithel, S. K., Maloney, S., Winston, C., Gönen, M., D’Angelica, M. I., Dematteo, R. P., et al. 2008. "Preoperative CA 19-9 and the Yield of Staging Laparoscopy in Patients with Radiographically Resectable Pancreatic Adenocarcinoma."Ann. SurgOncol. 15 (12): 3512-20.

[15] Karachristos, A., Scarmeas, N., and Hoffman J. P. 2005. "CA 19-9 Levels Predict Results of Staging Laparoscopy in Pancreatic Cancer." J. Gastrointest Surg. 9 (9): 1286-92.

[16] Fujioka, S., Misawa, T., Okamoto, T., Gocho, T., Futagawa, Y., Ishida, Y., et al. 2007. "Preoperative Serum Carcinoembryonic Antigen and Carbohydrate Antigen 19-9 Levels for the Evaluation of Curability and Resectability in Patients with Pancreatic Adenocarcinoma." J. Hepatobiliary Pancreat Surg. 14 (6): 539-44.

[17] Kiliç, M., Göçmen, E., Tez, M., Ertan, T., Keskek, M., and Mahmut, K. 2006. "Value of Preoperative Serum CA 19-9 Levels in Predicting Resectability for Pancreatic Cancer." Can. J. Surg. 49 (4): 241.

[18] Goldstein, D., El-Maraghi, R. H., Hammel, P., Heinemann, V., Kunzmann, V., Sastre, J., et al. 2015. "nab-Paclitaxel Plus Gemcitabine for Metastatic Pancreatic Cancer: Long-Term Survival from a Phase III Trial." J. Natl. Cancer Inst. 107 (2): dju413.

[19] Lewis, R., Drebin, J. A., Callery, M. P., Fraker, D., Kent, T. S., Gates, J., et al. 2013. "A Contemporary Analysis of Survival for Resected Pancreatic Ductal Adenocarcinoma." HPB (Oxford) 15 (1): 49-60. 